\title{
Pour une meilleure performance individuelle au travail
}

\author{
Ellioua Hanane \\ Hassan First University of Settat, ENSA Berrechid, Morocco
}

\begin{abstract}
Résumé: Dans un contexte où les organisations font face à plusieurs changements, tels que la concurrence accrue, l'évolution technologique, l'accroissement des impératifs de qualité, de coûts et de délais dans la livraison des produits et des services, les dirigeants sont appelés à revoir le mode d'organisation du travail, La performance individuelle au travail constitue une problématique qui a non seulement permis aux entreprises du monde entier de se faire une place, mais qui a également alimenté de nombreuses recherches dans les domaines de la gestion, de la santé au travail et de la psychologie de l'organisation. Malgré l'importance de ce construit, des recherches dans le domaine ont montré qu'il n'y a pas de consensus sur sa définition et sa mesure. L'objectif de ce travail est de développer un état de l'art sur la performance professionnelle des individus en s'intéressant à ses déterminants et à ses indicateurs de mesure.
\end{abstract}

Mots-clés : Performance, Performance individuelle, performance au travail, dimensions de la performance, mesure de la performance.

\section{INTRODUCTION}

Notre environnement est toujours en perpétuel changeant ainsi que l'instabilité économique ces dernières années ont forcé les entreprises de reconsidérer la place du personnel dans leurs organisations, ainsi que l'importance non négligeable des individus dans le développement de la vie économique mondiale. En effet, toute entreprise de tout secteur confondu et toute taille, prend en conscience du constat disant que le personnel est un facteur clé de sucées pour l'entreprise. Historiquement et plus précisément au milieu du 20ème siècle, le modèle taylorien a privilégié la réalité qu'a connu la fonction du personnel au sein des organisations ou il n'y avait que des ouvriers exécutant la même tâche toute leur vie et cela sans aucune considérations ou reconnaissance à leur égard.

La fonction du personnel s'est influencée par le système productif sur lequel s'appuie la gestion de l'entreprise, donc elle s'est changée à la gestion des ressources humaines en recouvrant plus des activités et plus de responsabilités. La gestion des ressources humaines a connu plusieurs évolutions au cours du temps, l'ensemble des missions que recouvre actuellement est bien plus large et bien plus complexe qu'à son origine. Cela est expliqué d'une part par les changements qu'a connu l'environnement de l'entreprise tels que les changements économiques, technologiques ou sociales. D'autre part, les travaux élaborés par les différents chercheurs dans le domaine ont influencé également la place de la fonction de la gestion des ressources humaines au sein de l'organisation.

La littérature à travers plusieurs chercheurs atteste que les ressources humaines représentent un avantage concurrentiel pour l'organisation. En effet, une panoplie des auteurs ont mis le point sur le fait que la principale source d'avantages compétitifs est le capital humain comme Miller (1989) qui a souligné que « Les ressources humains procurent à l'organisation un avantage compétitif. ». Empiriquement, des chercheurs tels que, Delery 
et Doty (1996), Arcand (2000), Liouville et Bayad (1995) et Huselid (1995), Lacoursière (2001), soutiennent totalement cette vision que la gestion des ressources humaines est une fonction qui favorise la rentabilité financière de l'entreprise.

La gestion des ressources humaines était depuis longtemps considérée l'une des activités qui servent à supporter les autres fonctions de l'organisation, aujourd'hui elle représente une source d'avantage concurrentiel à long terme. Et cela à travers l'amélioration des systèmes attachés à cette fonction tout en fixant un objectif d'attirer et de conserver une main d'ouvre compétente et motivée. St-Onge (2009) définit la gestion des ressources humaines comme «l'ensemble des pratiques de planification, d'organisation, de direction et de contrôle des ressources humaines, des employés d'une entreprise. » A cet égard nous pouvons identifier les pratiques de gestion des ressources humaines tels que la gestion de carrières, la formation, la motivation, la rémunération... au sein de l'organisation qui peuvent constituer le noyau majeur pour son développement. En se basant sur la définition déjà mentionnée, la gestion des ressources humaines peut être considérée comme une source précieuse qui pourrait être déterminante pour la pérennité d'une entreprise voire sa performance.

Partant de cette notion de la performance qui est considéré comme l'axe central pour évaluer les systèmes et démarches d'une organisation. Généralement la performance est définie comme « un constat officiel enregistrant un résultat accompli à un instant $t$, toujours en référence à un contexte, à un objectif et un résultat attendu, et ce quel que soit le domaine. » (Notat, 2007).

Pratiquement on déduit que la performance est un concept holistique n'admit pas à une seule définition mais il prend plusieurs formes selon le contexte où il est employé. Le rôle central de la performance individuelle dans le succès organisationnel nécessite de la part des entreprises une attention particulière quant à sa conceptualisation et à sa mesure. En effet, ce concept multidimensionnel connait encore un retard dans sa définition et dans la mise en place d'un système de mesure valide et équitable.

Ainsi, l'objectif du présent travail est de développer un cadre conceptuel de la performance individuelle au travail en répondant au questionnement suivant : Comment peut-on définir la performance individuelle au travail ? Quels sont ses déterminants et ses dimensions ? Et surtout comment mesurer et appréhender la performance professionnelle des individus?

Pour répondre à cette problématique nous allons d'abord nous intéresser aux différentes définitions et aux modèles théoriques développés puis en distinguant ses différentes dimensions et ses déterminants individuels et organisationnels. Enfin, nous allons essayer de proposer des indicateurs de mesure de la performance puisqu'il n'existe toujours pas un système générique de sa mesure.

\section{LA PERFORMANCE AU TRAVAIL : REVUE DE LITTERATURE}

Malgré la grande pertinence de la performance individuelle et son utilisation généralisée comme mesure de résultat à la fois dans les milieux académiques et professionnels, il s'avère que peu d'efforts ont été consacrés à la clarification de ce concept. Il s'agit en effet d'un construit complexe relevant de plusieurs disciplines et revêtant plusieurs dimensions d'où son caractère polysémique (Idrissi \& Loulid, 2018).

\section{Définition de la performance individuelle au travail:}

La performance au travail est un concept multidimensionnel. Sur le plan basique, on peut distinguer entre un aspect processus et un aspect résultat. L'aspect processus renvoie à « ce que les gens font au travail, à l'action elle-même » (Volmer et al, 2008 : 427). Cependant, la performance n'est pas définie par l'action elle-même, mais par le jugement et le processus évaluatif (Sonnentag \& Frese, 2005). Par ailleurs, seules les actions qui peuvent être hiérarchisées et mesurées sont considérées dans la constitution de la performance (Campbell et al, 1993). L'aspect résultat, quant à lui, désigne la conséquence du comportement de l'individu (l'action menée). (Volmer et al, 2008). 
Campbell (1990 : 40) affirme que la « performance est ce que l'organisation recrute un individu pour faire et le faire bien ». Il définit la performance comme les actions ou les comportements sous le contrôle de l'individu, qui contribuent aux objectifs de l'organisation, et qui peuvent être mesurés en fonction du niveau de la compétence de l'individu. Ainsi, « La performance au travail est définie comme la valeur totale attendue par l'organisation des épisodes comportementaux discrets qu'un individu effectue sur une période de temps donnée » (Motowildo, 2003: 39). Nous pouvons donc constater que la performance correspond à l'ensemble des comportements des individus visent à améliorer l'efficacité au travail sur des périodes de temps variées.

La performance individuelle peut être considérée comme « une activité dans laquelle un individu est capable d'accomplir avec succès la tâche qui lui est assignée, sous réserve des contraintes normales de l'utilisation raisonnable des ressources disponibles » (Jamal, 2016). Cette définition, plus développée, met l'accent sur les deux composantes principales de la performance ; l'efficacité et l'efficience. Ce qui implique que la performance correspond à l'atteinte des objectifs tout en faisant bon usage des moyens déployés par l'organisation pour les atteindre. Murphy et Cleveland (1995) estiment que la performance constitue l'ensemble des comportements qui sont pertinents pour l'organisation dans laquelle une personne travaille. Rubina et al. (2008) ont défini la performance au travail comme étant le résultat de trois facteurs qui agissent ensemble : la compétence, l'effort et la nature des conditions de travail. Les compétences comprennent les connaissances, les capacités et les compétences des employés ; l'effort est le degré de motivation que l'employé met en avant pour terminer le travail et la nature des conditions de travail est le degré d'accommodement de ces conditions pour faciliter la performance de l'employé. Le concept de la performance n'admet pas une seule acception vu les différentes dimensions qui le composent. La littérature sur la performance au travail a été marquée par deux grands modèles qui seront présentés ci-après.

\section{LES DETERMINANTS PERSONNELS DE LA PERFORMANCE AU TRAVAIL}

Sur le plan individuel, Campbell (1990) distingue entre trois grands déterminants de la performance : les connaissances déclaratives, les connaissances et compétences procédurales et la motivation. La connaissance déclarative comprend la connaissance des faits, des principes, des buts et du soi. Elle est supposée être fonction des capacités, de la personnalité, des intérêts, de l'éducation, de la formation, de l'expérience et des interactions aptitude- traitement d'une personne. Les connaissances et compétences procédurales comprennent les habiletés cognitives et psychomotrices, les habiletés physiques, les compétences en gestion de soi et les compétences interpersonnelles. Les prédicteurs des connaissances et des compétences procédurales sont à nouveau les capacités, la personnalité, les intérêts, l'éducation, la formation, l'expérience et les interactions entre l'aptitude et le traitement, ainsi que la pratique. La motivation comprend le choix à effectuer, le niveau d'effort et la persistance de l'effort. Les différences individuelles en termes de motivation peuvent être dues à des différences dans les traits de motivation mais aussi à des différences dans les compétences de motivation (Kanfer \& Heggestad, 1997). Les traits de motivation sont étroitement liés aux constructions de personnalité, mais ils sont plus étroits et plus pertinents pour les processus de motivation, c'est-à-dire l'intensité et la persistance d'une action.

D'après Sonnentag et Frese (2005), il existe une relation forte entre la capacité cognitive et la performance au travail. Les personnes ayant des capacités cognitives élevées obtiennent de meilleurs résultats que les personnes ayant de faibles capacités cognitives dans un large éventail d'emplois différents. Ces auteurs supposent un mécanisme sous-jacent de capacité cognitive aidant à acquérir des connaissances et des compétences professionnelles qui ont un impact positif sur la performance au travail. Cette perspective des différences individuelles est intéressante à prendre en compte dans le recrutement du capital humain afin de maximiser la performance individuelle au travail. 
Les déterminants organisationnels de la performance au travail :

Contrairement aux déterminants individuels, la perspective organisationnelle de la performance renvoie aux facteurs du milieu du travail qui peuvent venir faciliter ou freiner la performance des individus. Le modèle de Hackman \& Oldham (1976) constitue une bonne illustration de la première catégorie de facteurs, notamment ceux qui facilitent et encouragent la performance au travail. Dans ce modèle, Hackman et Oldham ont supposé que les caractéristiques du travail (La diversité des compétences, l'identité des tâches, l'importance des tâches, l'autonomie, le feedback) influent sur les états psychologiques critiques (sens de l'expérience, responsabilité éprouvée pour les résultats du travail, connaissance des résultats des activités de travail) qui, à leur tour, ont un effet sur les résultats personnels et professionnels, y compris la performance au travail.

Les approches de la deuxième catégorie mettent l'accent sur les facteurs qui nuisent aux performances. Dans la théorie des rôles (Kahn et al, 1964), l'ambiguïté des rôles et les conflits de rôles sont conceptualisés comme des facteurs de stress. Ainsi, les contraintes organisationnelles comprennent les facteurs de stress tels que le manque d'informations nécessaires, les problèmes de machines et de fournitures ce qui impacte directement la performance au travail.

Sonnentag et Frese (2005) concluent que, dans une perspective organisationnelle, les facteurs d'amélioration de la performance (par exemple, le contrôle au travail, les tâches significatives) jouent un rôle plus important que les facteurs de stress. Autrement dit, le manque de caractéristiques positives dans la situation de travail telles que le contrôle au travail menace la performance plus que la présence de certains facteurs de stress. Les différences individuelles en termes de performance nécessitent la mise en place d'un système de mesure afin de déterminer les différents facteurs à incidence directe sur la performance.

\section{LES MODELES DE LA PERFORMANCE AU TRAVAIL}

La littérature sur la performance a été marquée par des modèles génériques applicables aux différents contextes comme par des modèles spécifiques à des domaines précis ; militaire ou sportif par exemple. Or, les principaux modèles ayant tenté de cerner la performance sous ses différentes facettes restent le modèle multifactoriel de Campbell (1990) et le modèle de Borman et Motowildo (1993).

\section{A. Le modèle multifactoriel de Campbell (1990) :}

Ce modèle a été développé pour la première fois dans le domaine militaire avant d'être revisité pour s'adapter aux différents contextes. Il s'agit de l'un des premiers modèles ayant abordé la performance en développant huit facteurs qui la constituent. Ces dimensions, ou facteurs, présentent trois grandes caractéristiques. Elles sont génériques, universelles et indépendantes (Charles-pauvers et al, 2007). Le tableau suivant résume ces différentes dimensions comportementales d'après Charles-pauvers et al (2007).

La première dimension comportementale sont les compétences dans les tâches spécifiques l'emploi, c'est le niveau de compétence avec lequel un individu exécute les tâches techniques spécifiques à son emploi, la deuxième dimension traite les compétences dans les tâches non spécifiques à l'emploi, cette dimension vise à maîtriser les tâches communes à plusieurs emplois de l'organisation. Cette aptitude relève des compétences à la polyvalence et à l'adaptabilité. La troisième dimension est La communication écrite et orale, elle vise à communiquer clairement et efficacement oralement, ou par écrit, la quatrième dimension sont les efforts au travail, elle vise à démontrer des efforts supplémentaires et à travailler efficacement dans des conditions difficiles (motivation à réaliser les tâches assignées avec énergie, intensité et persistance), la cinquième dimension est le maintien d'une discipline personnelle qui cherche à éviter les comportements négatifs ou contreproductifs (ex : infraction aux règles internes, absentéisme, manque de ponctualité), la sixième 
dimension est La facilitation de la performance de l'équipe et des collègues, cette dimension, relève de l'aptitude d'une personne à soutenir, à aider, et à développer les collègues et à concourir à l'unité de l'équipe en contribuant à son fonctionnement collectif. La septième dimension est la supervision qui vise à influencer les personnes subordonnées par des interactions en face à face. Cette compétence relève des qualités du leader exprimé par l'individu ou observées dans son art de manager une équipe, la dernière dimension est Le management et l'administration qui vise à maîtriser les tâches d'allocation des ressources, d'organisation, de contrôle de l'efficacité d'une équipe (contrôle et suivi des objectifs, de l'application des règles) et de développement d'un service (accroître les ressources humaines, financières, matérielles). La performance individuelle au travail peut donc être appréhendée par le biais de ces différents facteurs. Cette modélisation a constitué le point de départ pour d'autres modèles de la performance au travail dont le plus important reste celui de Borman et Motowildo (1993).

\section{B. Le modèle bidimensionnel de Borman et Motowildo (1993)}

Ce deuxième modèle distingue entre deux dimensions essentielles quant à la composition de la performance individuelle. Il s'agit de la performance dans la tâche et de la performance contextuelle.

Motowildo (2003 : 46) définit la performance dans la tâche comme étant « la valeur totale attendue des comportements d'un individu sur une période de temps pour la production des biens ou des services de l'organisation ». Cette performance est relative aux différentes tâches réalisées par les individus en vue d'atteindre des objectifs prescrits. La performance contextuelle quant à elle, renvoie à la valeur totale attendue des comportements d'un individu sur une période de temps pour maintenir et améliorer le contexte psychologique, social et organisationnel du travail (Motowildo, 2003). L'objectif de cette deuxième dimension est, donc, d'améliorer l'efficacité et l'efficience organisationnelles par la promotion de comportements productifs dans les milieux de travail.

Borman et Motowildo (1993 ; 1997) distinguent entre cinq dimensions de la performance contextuelle (Charbonnier \& Silva, 2007) :

- Persister avec enthousiasme et déployer des efforts pour accomplir ses tâches avec succès ;

- S'engager volontairement dans des tâches et activités qui ne font pas formellement partie de son travail ;

- Aider et coopérer avec les autres ;

- Suivre les règles et les procédures organisationnelles ;

- Approuver, défendre et soutenir sincèrement les objectifs organisationnels.

\section{LES DIMENSIONS DE LA PERFORMANCE INDIVIDUELLE AU TRAVAIL}

De nos jours, la littérature distingue entre quatre grandes dimensions de la performance au travail. Outre la performance dans la tâche et contextuelle telles que développées précédemment, deux autres dimensions sont venues caractériser la performance des individus : la performance adaptative et les comportements contreproductifs.

L'attention portée au comportement contreproductif, défini comme un comportement nuisible au bien-être de l'organisation, a augmenté au cours des dernières années. Il inclut des comportements tels que l'absentéisme, le retard au travail, le comportement hors travail, le vol et la toxicomanie (Koopmans et al, 2011). Rotundo et Sackett (2002) ont conclu que le comportement contre-productif devrait être distingué comme la troisième grande dimension de la performance individuelle au travail (en plus de la performance dans la tâche et contextuelle). L'intérêt porté à cette dimension se justifie par la nécessité de repérer les comportements 
susceptibles de freiner ou de retarder la performance au travail. C'est comportements constituent un dysfonctionnement qui nuit aux performances humaine et organisationnelle.

La dernière dimension de la performance renvoie à celle adaptative. Griffin et Parker (2007) la définissent comme étant la capacité d'un employé à s'adapter aux changements dans les rôles de travail ou dans le système de travail. Cette dimension adaptative n'a pas fait l'objet des modélisations antérieures de la performance au travail. Or, en raison des environnements de travail changeants et dynamiques, le besoin en employés adaptatifs est devenu de plus en plus important (Pulakos et al., 2000 ; Smith et al., 1997).

Pulakos et al. (2000) ont distingué entre huit dimensions de la performance adaptative :

- Gérer les urgences ou les situations de crise ;

- Gérer le stress au travail ;

- Résoudre les problèmes de façon créative ;

- Faire face à des situations de travail incertaines et imprévisibles ;

- Apprendre les tâches, les technologies et les procédures de travail ;

- Démontrer une capacité d'adaptation interpersonnelle ;

- Démontrer une adaptabilité culturelle ;

- Démontrer une adaptabilité physique.

Ces auteurs ont démontré que les différentes dimensions de la performance adaptative sont présentes dans de nombreux types d'emploi (Volmer et al, 2008) d'où leur caractéristique générique.

Les différentes dimensions restent complémentaires dans la définition de la performance des individus au travail. Cependant, une meilleure compréhension de ce concept nécessite de définir ses antécédents qu'ils soient liés à la personne ou au travail.

\section{CONCLUSION}

Les changements dans les environnements de travail n'ont pas été sans effet sur la performance des organisations. De nos jours, la performance des entreprises ne dépend plus de celle financière mais s'est désormais élargit pour tenir compte de la dimension humaine. Ainsi, le capital humain constitue la vraie richesse d'une organisation dont il est important d'appréhender le niveau de performance afin de développer un avantage compétitif. Malgré l'importance que revêt la performance des individus au travail, ce concept multidimensionnel est encore difficile à définir et par conséquent à mesurer. En effet, quatre grandes dimensions caractérisent la performance individuelle : la performance dans la tâche, la performance contextuelle, la performance adaptative et les comportements contreproductifs. Ainsi, le développement d'un système de mesure de la performance au travail peut s'inspirer des différents indicateurs relatifs à ces dimensions afin de concevoir une mesure unifiée et générique de la performance individuelle au travail, Le présent article dresse un cadre général de la performance, des travaux ultérieurs s'avèrent nécessaires afin de développer un modèle de la performance qui tient compte des différentes dimensions et qui peut être adopté dans la mesure de l'efficacité et l'efficience du capital humain d'une organisation. 


\section{BIBLIOGRAPHIE}

[1] Borman,W. C., \& Motowidlo, S. J. (1993). Expanding the criterion domain to include elements of contextual performance. New York: Jossey-Bass.

[2] Borman, W. C., \& Motowidlo, S. J. (1997). Task performance and contextual performance: The meaning for personnel selection research. Human Performance, 10,99-109.

[3] J. Campbell, J. P. (1990). Modeling the performance prediction problem in industrial and organizational psychology. Palo Alto: Consulting Psychologists Press.

[4] Campbell, J. P., McCloy, R. A., Oppler, S. H., \& Sager, C. E. (1993). A theory of performance. San Francisco: Jossey-Bass.

[5] Charbonnier, A., Silva C.-A. \& Roussel P. (2007). Vers une mesure de la performance contextuelle au travail de l'individu : étude exploratoire, Actes du congrès de l'AGRH, fribourg.

[6] Charles-Pauvers, B., Commeiras, N., Peyrat-Guillard, D., \& Roussel, P. (2007). La performance au travail et ses déterminants psychologiques. De Boeck, collection Méthodes et Recherches.

[7] Gopal, K. Kanji (2002). Performance measurement system. Total Quality Management, 13(5), 715-728.

[8] Soumaya DLIMI (2015). The Importance of the Strategy Governance for the Preservation of the Performance of the Teams. American Journal of Business and Management.4.

[9] Griffin, M. A., Neal, A., \& Parker, S. K. (2007). A new model of work role performance: Positive behavior in uncertain and interdependent contexts. Academy of Management Journal, 50, 327-347.

[10] Hackman, J. R.,\&Oldham, G. R. (1976). Motivation through the design ofwork: Test of a theory. Organizational Behavior and Human Performance, 16, 250-279.

[11] Idrissi, N. \& Loulid, A. (2018). Effet des mécanismes de gouvernance sur la performance des sociétés marocaines cotées en bourse. Revue du Contrôle de la Comptabilité et de l'Audit, 4, 97-107.

[12] Jamal, M. (2016). Job Stress and Job Performance Relationship in Challenge-Hindrance Model of Stress: An Empirical Examination in the Middle East , Pakistan Journal of Commerce and Social Sciences, 10(3), 404-418.

[13] Kahn, R. L.,Wolfe, D. M., Quinn, R. P., Snoek, J. D., \& Rosenthal, R. A. (1964). Organizational stress: Studies in role conflict and ambiguity. New York:Wil. 